\section{ROBUSTIANA ARMIÑO, LA MODERADA EXALTACIÓN}

\author{
Bernardino M. Hernando \\ Asociación de la Prensa de Madrid \\ bmhernando@apmadrid.es
}

\section{ROBUSTIANA ARMIÑO, THE MODERATE EXALTATION}

Cómo citar este artículo/Citation: Hernando, B. M. (2014). “Robustiana Armiño, la moderada exaltación". Arbor, 190 (767): a139. doi: http://dx.doi.org/10.3989/arbor.2014.767n3010
Copyright: (c) 2014 CSIC. Este es un artículo de acceso abierto distribuido bajo los términos de la licencia Creative Commons Attribution-Non Commercial (by-nc) Spain 3.0.

Recibido: 3 julio 2013. Aceptado: 6 abril 2014.

RESUMEN: Hay un mundo literario español tan intenso y vivo como poco conocido: el de las mujeres escritoras de la segunda mitad del siglo XIX. Apenas un puñado exquisito, considerado de primera fila, logró pasar a la historia de los tratados y las antologías. Ponemos nuestra atención en una mujer escritora de esa época, Robustiana Armiño, considerada de segunda fila, lo que viene a equivaler casi a la nada en cuanto a la resonancia de su obra desarrollada principalmente, como la de la mayoría, en los periódicos. La escritora y periodista de insólito nombre merece alguna atención, mayor de la que ha tenido.

PALABRAS CLAVE: Robustiana Armiño; mujeres periodistas españolas; periodismo español del siglo XIX.
ABSTRACT: There is a Spanish literary world that is as intense and alive as it is little known: that of women writers of the second half of the 19th century. A mere handful, regarded to be of the highest rank, managed to go on to the history of treatises and anthologies. We turn our attention here to a woman writer of this epoch: Robustiana Armiño, viewed as a second-rank writer, which meant her work, mostly published in newspapers, has passed almost unnoticed. However, this unusually named writer and journalist deserves more attention than she has usually received.
KEYWORDS: Robustiana Armiño; women Spanish journalists; C19th Spanish journalism. 
Robustiana Armiño (Gijón, 1821-Madrid, 1890) es una escritora casi desconocida (1) o, como delicadamente dice la investigadora asturiana María Elvira Muñiz Martín, "una decorosa figura de segundo orden en la literatura de la época" (Muñiz Martín, 1990).

Sobre Robustiana Armiño, la única escritora española que se llama Robustiana, se han repetido algunos tópicos que debemos matizar aquí. Carolina Coronado escribió el prólogo al primer tomo de sus poesías (1851) en el que dibuja un perfil que todo el mundo parece haber dado por bueno. Después de referirse a la "Señorita Doña Robustiana Armiño", con amistosa y romántica desmesura, como "la más alta joya de las Asturias, ídolo de Gijón”, pinta un cuadro a medias glorioso a medias fúnebre. La Armiño sería una bondadosísima mujer prudente y equilibrada, instruida y autodidacta, pero sumergida en la niebla de la tristeza y el desencanto. Tener que compaginar sus anhelos poéticos con la dureza de la realidad doméstica y social hubieron de llevarla a una tristeza irremediable. Coronado habla de la "juiciosa ambición" de R. A., que renunció a ser más grande como artista para ser grande como mujer, esposa y madre.

Sin embargo, la vida de Robustiana Armiño no se distinguió mucho de la de sus contemporáneas ni sus poesías rezuman ninguna tristeza especial que no estuviera en la entraña romántica. Y el hecho de colocar el apellido de su marido como segundo suyo (Robustiana Armiño de Cuesta) solo respondía a una equívoca costumbre que ha perdurado hasta hoy y que ella siguió solo a veces. Y tuvo una vida familiar más satisfactoria que la de la mayoría.

Otro tópico llamativo es el de su supuesta ideología encarnizadamente carlista, por la que ha recibido durísimos adjetivos de repulsa (2).

Su carlismo se habría reflejado en frecuentes artículos publicados en La Esperanza, Altar y Trono y otras publicaciones carlistas. Pero en La Esperanza jamás publicó artículo alguno (sí, en cambio, su amigo Plácido Jove y Hevia) y en Altar y Trono solo publicó un breve texto dedicado a Jerusalén (tema recurrente en su obra periodística, lo que alimenta la sospecha de algún viaje real a la ciudad: “...presenta a los ojos del viajero que la saluda por primera vez") (3).

Por fin, el tópico mayor es considerar a Robustiana Armiño mera poetisa (Miralles, 2006, pp. 17-18 y pp. 173-181), cuando sus publicaciones poéticas tuvieron fecha de caducidad y la inmensa mayoría de sus obras fueron artículos para los periódicos y no en función de simple colaboradora. De modo que si hoy hubiera que restringir su carácter de escritora a una sola función (que, por supuesto, no hay por qué) habría que etiquetarla de periodista.

\section{EL ENIGMA DE UN NOMBRE PINTORESCO}

Robustiana no es nombre muy común. Tampoco Armiño es apellido frecuente. Juntos huelen a seudónimo (4). Y en realidad es, técnicamente, un seudónimo.

Su nombre completo, según la partida de bautismo (5), es Robustiana Josefa Antonia Ángela Alonso de Armiño Menéndez, que "se bautizó en esta iglesia parroquial de San Pedro de Gijón, el 21 de agosto de 1821, habiendo nacido el día anterior". En la misma iglesia son bautizados los demás hermanos: Dorotea (1823), Antonino (1826) y Antonino Demetrio (1830), hijos del farmacéutico don Buenaventura Alonso de Armiño de Ruboca y Dña. María Menéndez y Fernández Muñiz. La repetición del nombre de Antonino en los dos hermanos se debería a la muerte del primero, de la que queda constancia en los versos de Robustiana ("En la tumba de mi hermano Antonino", en el Album, al que enseguida haremos referencia).

El nombre de Robustiana no es eufónico ni elegante, ni casi de calendario católico (¿hubo alguna santa Robustiana?; lo que hubo es un San Robustiano y con eso basta) pero con ese sabor a seudónimo no queda mal como reclamo.

Más curioso resulta la supresión del primer elemento del apellido compuesto, parece que de algún abolengo histórico, "Alonso de Armiño". La preferencia familiar por la fina rotundidez del suave "Armiño" en lugar del apellido completo no deja de ser sugestiva (6).

\section{LA CIUDAD DE GIJÓN}

Gijón, en 1821, año del nacimiento de Robustiana Armiño, tenía poco más de 7.000 habitantes y una vida social y cultural aún más modesta que la de 40 años después, cuando el asturiano Armando Palacio Valdés la representó en su novela El cuarto poder, con el nombre de Sarrió (7).

Sin embargo, en Sarrió-Gijón hay vida social y cultural. Como la hay en el Gijón de Robustiana, cuyos comienzos culturales se mueven en dos ámbitos, el familiar y el social-amistoso.

Quizá la primera chispa le llegara desde la tertulia que, posiblemente, su padre mantuviera en la rebotica, una tradición ineludible en aquella época (8). Sobre todo si se tiene en cuenta el nivel de interés de la familia (9). El Album romanticu de Dorotea Armiño, 
la hermana de Robustiana (Cano González, 2002) es revelador de un cierto elitismo.

De los cuatro hermanos Armiño son Robustiana y Dorotea (a la que a veces se llama "Dora", ¿cómo llamarían a Robustiana?) las que fraguaron una relación más estrecha e íntima, como acreditan los versos dedicados por la primera a la segunda y los poemas dedicados a Dorotea en ninguno de los cuales se deja de citar a Robustiana en la misma onda de intenso afecto. Dorotea es sensible a la poesía, pero ella practica el dibujo artístico (también lo practica, aunque en menor medida, Robustiana) y de todo ello, y de las aficiones culturales de la familia, hay ejemplos en el Album: de las inclinaciones artísticas y poéticas de Juan Cuesta y del dominio de Robustiana Armiño en el manejo poético del idioma francés. Añadamos que el hermano menor (1830) se hizo bachiller en Letras por la Facultad de Filosofía de Oviedo (1841-1846) y estudió Farmacia sin llegar a terminarla, y fue regente de Farmacia al mismo tiempo que colaboraba en prensa.

Esta trama intelectual familiar tuvo en sus años gijoneses, hasta 1848 en que se casa con Juan Cuesta y se traslada con él a su tierra salmantina, otro entramado amistoso que le sirvió para construir las bases esforzadas de una cultura que más adelante sería admirada y alabada por muchos. Sin los nombres amigos de Eulalia de Llanos y Noriega, de Plácido Jove y Hevia y de Gumersindo Laverde, no se entendería la personalidad de Robustiana Armiño (10). Además de la influencia del citado Juan Cuesta, médico con aficiones literarias, natural de Salamanca, que casó con $\mathrm{R}$. A. cuando esta tenía 27 años (1848), edad avanzada para la época y que la había colocado en el ingrato papel de "moza vieja", según la terminología social de entonces, que hoy nos hace sonreír (la Baronesa de Wilson casó a los 15 años; la Pardo Bazán, a los 16; Vicenta García Miranda, a los 17... Isabel II, a los 16, pero ese es otro cantar y muy otro cantar es la madurez a la que casaron Gertrudis Gómez de Avellaneda y Carolina Coronado, a los 32 años).

En sus años gijoneses se pone en contacto con Carolina Coronado, a través de la cual establece amistosa relación con escritores y escritoras (Víctor Balaguer, J.E. Hartzenbusch, Gertrudis Gómez de Avellaneda...). En 1844, Carolina Coronado convoca a algunas escritoras para colaborar en El Pensamiento (periódico) y en El Ateneo de Badajoz, que un año antes había fundado su hermano Pedro. Entre ellas, Robustiana Armiño y la extremeña Vicenta García Miranda son nombradas Socias Facultativas del Liceo de Badajoz, título tan hermoso como inútil. Vicenta García Miran- da (1816-1877), hija de farmacéutico y casada con un médico, exactamente igual que Robustiana Armiño, pero de vida dolorosa, escribiría ilusionada:

"iAh! ¿por qué percibieron mis oídos/Oh Carolina y Robustiana hermosas,/De vuestras dulces liras los sonidos?

Robustiana Armiño, con los estudios escolares elementales comunes en la época (su novela Dramas de la costa, 1879, da luz sobre la educación escolar de entonces) se propuso y consiguió por su cuenta y riesgo tres cosas: una cultura respetable, un respetable dominio de la versificación y el manejo con la soltura necesaria para leer y escribir, y aun traducir, en (además del español, el gallego y el bable) francés, inglés, alemán e italiano (11). De que todo eso fue muy difícil queda constancia en los dos poemas que resumen su vida en Gijón: "Recuerdos de mi patria" y “Despedida a Gijón”, que incluiría en el tomo I de sus poesías (12).

En "Despedida a Gijón" muestra su ilusión por ir en busca de "nuevas ilusiones", "en dulce compañía"; abandona Gijón donde ha sufrido "niñez amarga" aunque también guarda el recuerdo "de bellos días y pasadas glorias" y deja "un tesoro, más precioso que el oro, la joya más hermosa de mi vida, mi familia querida". Y hace una referencia, tan romántica como realista, al duro, solitario aprendizaje de idiomas:

¡Adiós hogar paterno;

Dó en la noche sombría

Del nebuloso invierno,

Sobre áspera gramática inclinada,

Iba con alma osada,

Sin maestro ni guía

Los idiomas del norte descifrando,

Al son del ronco viento

Que estaba mis cristales azotando.

Las hermanas Armiño, Robustiana y Dorotea, estaban tan unidas que el gran dolor de la separación, como dice en "Recuerdos de mi patria" ("iAy! triste, solitaria- quedó la pobre tórtola en su nido,-y la eterna plegaria- de un pecho dolorido-vibra incesante en mi turbado oído.-No, hermana querida- las horas riegues con amargo llanto- de tu edad más florida;-y escucha el triste canto- de un joven corazón que te ama tanto..) solo queda compensado por el amor del marido ("él a mi amante corazón consuela-él calma mis enojos-él enjuga mis ojos-él incesante por mi dicha vela...), y si parece triste, solo se debe a "la ausencia de la patria 
mía": echa de menos la frescura de los campos y los montes asturianos, y el mar, sobre todo el mar, frente a la sequedad castellana. (En Gijón, el 11 de mayo de 1990, le fue dedicada una calle. Nunca es tarde).

\section{EN TIERRA SALMANTINA}

Vive en Salamanca hasta 1859 y, además de disfrutar de un entorno cultural más elevado y dejar huella poética de sus vivencias ("A la catedral de Salamanca"), cuenta con una nueva amiga muy especial: la prima de su marido, Matilde Cherner (Rodríguez Sánchez, 2000; Rivas Arranz, 2013), cuyas actividades literarias, sociales y políticas (federalista militante) insuflan un ramalazo de aire nuevo en la vida de Robustiana Armiño (13), cuya moderación dejó siempre de lado compromisos políticos y exaltaciones de cualquier tipo. Le tocó vivir una época de enorme viveza política y social pasando por ella casi sin romperse ni mancharse, sobre todo en su actividad periodística. Neutral, a veces neutra, entregándose con fervor a intereses culturales, encarnándose, de momento, en la cultura salmantina, como demuestra su coautoría, con la Cherner, de La venganza, leyenda de las tierras de Las Arribes del Duero, patria chica de Matilde.

Y una vez más queremos valorar la importancia de Juan Cuesta Cherner en la vida de Robustiana Armiño, y viceversa, tanto en lo sentimental como en lo intelectual. Juan Cuesta no solo funda y dirige revistas de medicina, escribe poesía y otros textos literarios (la propia Robustiana se hace eco conmovido del libro Días geniales (cf. "Fotografías sociales", pág. 248) y comenta con amor y ternura otros aspectos del marido... (14).

Se puede hablar, pues, de dos grupos de amigos: el grupo de Gijón, ya citado, y el de Salamanca, al que pertenecen la prima Matilde Cherner y el salmantino Ventura Ruiz Aguilera (1820-1881), desterrado en Alicante, donde dirige Los Hijos de Eva (1849), en el que colabora Robustiana Armiño.

\section{LA CIUDAD DEFINITIVA}

Donde más años vivió Robustiana Armiño fue en Madrid:31 años. Cuando en 1859, a los 38 de edad, llega a Madrid con su marido y sus hijos, Juan, Favila, Antonio y Antonino, es moderadamente conocida, por sus dos tomos de poesías publicados viviendo en Salamanca, en 1851, y sus colaboraciones en periódicos de Madrid. Ya no es aquella tímida y siempre un poco insegura gijonesa. Que ya es conocida y reconocida en Madrid lo demuestra la invitación que recibe para colaborar en una "corona poética" en honor de Manuel José Quintana (15), junto a Hartzenbusch, Bécquer, Alarcón, López de Ayala, Ángela Grassi, María del Pilar Sinués y otros. La amistad con Carolina Coronado, de tan amplia e intensa vida social y las relaciones madrileñas de su esposo Juan Cuesta, médico, periodista especializado, editor y escritor, sentaron las bases para una nueva vida cultural y social.

Juan Cuesta muere en 1886 y ello explica, entre otras circunstancias, el retiro social en que Robustiana Armiño pasa los últimos años de su vida. Cuando muere en 1890, a los 69 de edad, se cierra una muy rica actividad de vida madrileña en la que prima el periodismo. Primero, porque edita y dirige un semanario, Ecos del Auseva, luego convertido en La Familia (1864-1869), de notable proyección social, al que estaba suscrita la reina Isabel II; y luego, porque llegó a ser mucho más que colaboradora en periódicos como La Aurora de la Vida (1861), Los Niños y El Correo de la Moda (1872), y sobre todo, El Periódico para todos (1872-1874), donde ejerció tareas de redacción y un estilo entonces poco frecuente, tanto en la elección de los temas como en su tratamiento. La inmensa mayoría de estos textos siguen durmiendo el sueño de las hemerotecas.

Se ha hablado mucho de sus obligaciones familiares difícilmente conciliables con su vocación literaria, sin tener en cuenta que su familia fue uno de sus apoyos fundamentales. Además de su culto y activo marido, Juan Cuesta, su hijo Favila colabora con el padre en tareas periodísticas y librescas; su hijo Juan dirige EI Propagador del Comercio y de la Industria" (1888).

\section{OBRA LITERARIA Y PERIODÍSTICA}

A partir de 1860, Robustiana Armiño apenas publica poesía. Y esa poesía solo da una imagen muy parcial suya; su más amplia representación está en los dos tomos editados en Oviedo en 1851, cuando ella vivía en Salamanca. Transmite el mundo netamente romántico: ilusiones y tristezas de la vida, monumentos y leyendas, personajes...y entre ellos, los poetas Quintana, Lista, Zorrilla, Lamartine..., con algunos ecos sorprendentes de San Juan de la Cruz y Fray Luis de León.

Su poesía es "de época", sentimental y moderada, de notable perfección formal y de gusto por la naturaleza. Gratifica su lectura pero, efectivamente, pertenece a "una decorosa figura de segundo orden".

El mismo decoroso lugar que ocupa como novelista: su novela principal, Dramas de la Costa (publicada por entregas en La Ilustración Gallega y Asturiana, 
en 1879) tiene el supremo encanto de constituir una mezcla bien conjuntada de romanticismo y realismo, justo cuando en España muere el romanticismo y nace el realismo, que llegará a erigirse en naturalismo. Dramas de la costa es una buena representación del movedizo momento novelesco español. Eso, el retrato social y religioso de una época con atisbos de osadía intelectual que son muy de agradecer y poco más... Que no es tan poco, según se mire.

De los textos dramáticos que se le atribuyen (el drama Los condes de Gijón, la zarzuela Dos coronas) no conocemos ninguno.

Otras publicaciones, algunas procedentes de periódicos, tienen marcado carácter patriótico, educativo y religioso. Comparables a las obras paralelas de sus contemporáneas, pero sin rayar a alturas superiores.

Es en los periódicos donde Robustiana Armiño publica casi toda su obra, aunque hay que distinguir la obra literaria (poesía, narraciones) de la netamente periodística (artículos, reportajes, sueltos). María del Carmen Simón Palmer, en su admirable Escritoras españolas del siglo XIX (Simón Palmer, 1991, pp. 61-65) hace una distribución de las colaboraciones periodísticas de nuestra autora por periódicos. Nosotros la hacemos por fechas, siguiendo su pauta y completándola en algunos casos. En cada uno de los periódicos indicamos, cuando nos es posible, el número de colaboraciones y su carácter (poemas, artículos...), así como algún nombre amigo que participaba en el mismo periódico.

Robustiana Armiño es, sobre todo, periodista. Murió cinco años antes de que se fundara la Asociación de la Prensa de Madrid. De haber vivido, dada su dedicación y relaciones profesionales, nos parece que habría pertenecido al grupo de sus fundadores. Lo que no deja de ser un futurible de razonable alivio sentimental.
(1) El 4 de julio de 1998, RTVE nos sorprendió con la serie "Mujeres en la historia. Las románticas", dedicada a Gertrudis Gómez de Avellaneda, Carolina Coronado y... Robustiana Armiño. En su tiempo no solo era conocida sino muy valorada (Parada, 1881 , p. 260 , p. 278 , p. 283 Criado y Domínguez, 1889, p. 51, p. 70, p. 71).

(2) "Pocos y sospechosos vasallos literarios le quedaban a Isabelona en los amenes de su reinado. Entre ellos la beatona -al poco furibunda carlista- Doña Robustiana Armiño de la Cuesta quien le dedicaba en 1864 su indigesta obra El Angel de los tristes..." (Ara Torralba, 2004, p. 273).

(3) Altar y Trono, Madrid, Año II-tomo IV, № 93, 5 de abril de 1871, pp. 348-349.

(4) Ese olor a seudónimo dio lugar a alguna anécdota muy divertida. El periodista mexicano Andrés Henestrosa (19062008) cuenta cómo el nombre que firma dos artículos en sendos periódicos mexicanos ("La Pascua de Resurrección en Oriente y Occidente", en El Federalista-1874- y “El judío errante", en El Mun- do -1899-) puede ser un seudónimo del gran jurisconsulto, escritor, político y diplomático Justo Sierra (1848-1912), que murió en Madrid como embajador de México. El nombre que firma esos artículos es el de Robustiana Armiño. Ambos habían sido publicados mucho antes en periódicos españoles.

(5) Datos tomados de Cano González, 2002, pp. 8 y ss.

(6) Un escritor actual, Mauro Armiño, también ha prescindido del primer elemento: se llama por partida legal Mauro Fernández Alonso de Armiño.

(7) Sarrió es el nombre que el novelista da a la ciudad donde se desarrolla la trama de El cuarto poder y que representa a Gijón: "Sarrió, hay que confesarlo de una vez, era una población dormida para todas las grandes manifestaciones del espíritu, para todas las luchas regeneradoras de la sociedad contemporánea. Nadie estudiaba los altos problemas de la política. Las terribles batallas que los diversos bandos libran para conseguir la victoria y el Poder no apasionaban en modo alguno los ánimos. En una palabra, en Sarrió, el año de gracia de 1860, no existía la vida pública. Se comía, se dormía, se trabajaba, se bailaba, se jugaba, se pagaba la contribución; pero todo de un modo absolutamente privado" (Palacio Valdés, 1918, pp. 60-70).

(8) Otro escritor asturiano, también hijo de boticario, Vital Aza (1898-1912) es autor del popular sainete La rebotica.

(9) Los estudios de farmacia ya habían alcanzado, por entonces, rango de estudios superiores. Los hermanos de Robustiana y, sobre todo, ella misma y su hermana Dorotea alimentaban evidentes fervores culturales.

(10) Eulalia de Llanos (1809?-1865). Colección de Composiciones poéticas de la señorita Doña Eulalia de Llanos y Noriega, publicadas por su hermana, señorita Doña Teresa, Gijón, Imp. y Lit. de Torre y Compañía, calle de la Libertad, no 82, 1871. A doña Robustiana Armiño:"No partas,Robustiana;-mira, no dejes los paternos lares-no dejes estos maresque brillan al albor de la mañana.-El 
manto azul de la serena Tetis- con su franja de plata..." (p. 272). El 10 de julio de 1992, Gijón dedicó una calle a Eulalia de Llanos.

Plácido Jove Hevia (1823-1909), Vizconde de Campo Grande, cónsul en Atenas, Nápoles, Malta, Perpiñán, Hamburgo, Lisboa y Argel, diputado, alto cargo en Hacienda, Agricultura y Sanidad, senador perpetuo, etc... Siendo estudiante, en 1842, funda en Gijón la Academia Artística y Literaria, a la que se unen Eulalia de Llanos y R. A. con quienes entabla una fuerte amistad. Cuando se va de Gijón, Robustiana Armiño le dedica un emocionado poema de despedida ("compañero de los bellos días", lo llama). "Uno de los vertebrados menos líricos y más sinuosos de la creación!", dijo de él Clarín con su característica mala rabia ("Palique", Madrid Cómico, no 444, 22-VIII-1891. Obras Completas, VIII, Artículos (1891-1894), p. 190.

Gumersindo Laverde (1835-1890), en $E$ I Álbum de la Juventud (dirigida por José Puente y Villanúa, catedrático de Literatura en Oviedo -luego en Zaragoza-, profesor de Laverde que, a su vez, es maestro y consejero de Menéndez Pelayo a quien recomienda algunos poemas de Robustiana Armiño. En 1851 comenta "Fotografías sociales" en El Eco de Salamanca. En sus Ensayos críticos sobre filosofía, literatura e instrucción pública españolas, Lugo, Imprenta Soto Freire, 1868, pp. 21-37, habla de R. A.

(11) Traduce del francés para El Correo de la Moda (1853, VIII, conclusión, №36, 30-IX-1853)) la biografía novelada "Marietta Tintoretta", de Eugenia Foa (1796185?) sobre Marietta Robusti, Ilamada
"Tintoretta" por ser hija de Jacopo Robusti, llamado Tintoretto (traducción firmada por una desconcertante Robustiana de Armiño Gómez, desconcierto que se repite en la firma de la dedicatoria de su libro Flores del Paraíso, donde igualmente escribe "Gómez". No acierto a explicar tan curiosa ¿errata?)

-Traduce del alemán "Cuentos de los Hermanos Grimm", para Los Niños, №13, noviembre de 1872.

En sus colaboraciones para El Periódico para todos traduce de periódicos ingleses y norteamericanos muchos textos que incorpora a sus artículos.

(12) Poesías de la señorita Dña. Robustiana Armiño. Sócia de mérito corresponsal del Liceo de La Habana, sócia facultativa del de Badajoz, ect. ect. Tomo primero. Oviedo, 1851, Imprenta y Litografía de Martínez Hermanos, pp.169-175. Hay tomo II.

(13) Matilde Cherner (1833-1880) nacida en Aldeávila de la Rivera (Salamanca) es autora de Cartas a Sofía en La llustración de la mujer (1875), Ocaso y Aurora, novela folletín en El Tiempo (Madrid), publicada en libro (Madrid, Sociedad de Tipógrafos, 1878) y María Magdalena. Estudio social (novela sobre la prostitución) (Madrid, Imprenta y fundación de la viuda e hijos de J. A. García, 1880). Murió en Madrid el 15 de agosto de 1880 "de muerte lamentable" (suicidio), según se dijo entonces, pero en realidad murió de un ataque cerebral. Usó el seudónimo de Rafael Luna. "Estudios sobre el Gil Blas (Lesage), del Suplemento al no 4 de La Ilustración Española y Americana, enero de
1875, pp.75-78. Conclusión en el no V 8-II-1875. "El Escudero Marcos de Obregón“, de Vicente Espinel

En Fotografías sociales, de Robustiana Armiño, coautora de "La venganza".

(14) Juan Cuesta Cherner (...-1886) es redactor de Anales de Beneficencia y Sanidad. Revista semanal científico-literaria (1860-1863) y director de La fuerza de un pensamiento. Revista mensual (18621863); funda, dirige y es propietario de La Correspondencia Médica. Periódico de noticias y de intereses materiales. Dedicado a los médicos, cirujanos, farmacéuticos, ministrantes y veterinarios de España (1864-1870); es autor de Días geniales (cuadros de costumbres), de La Serafina, juguete cómico en un acto y en verso, de poemas y traducciones del francés (Las aves, poema de Lamartine en Los hijos de Eva) y del prólogo al libro de Anastasio Perillán y García, Sucesos de la vida de un médico, Madrid, C.Baylly-Ballère, 1868. En 1876 financia la edición del Marcos de Obregón de Vicente Espinel, sobre el que escribiría su prima Matilde Cherner. Es autor, junto a su hijo Favila, de Código médico-farmacéutico español o recopilación de leyes, reglamentos, reales órdenes y disposiciones vigentes, relativas al ejercicio de la medicina y la farmacia (Madrid, Imprenta de G.Yuste, 1885).

(15) La coronación de Manuel José Quintana como poeta por la reina Isabel II, el 25 de marzo de 1855, fue un acontecimiento social de primer orden del que sirvieron de eco dos antologías ("coronas") poéticas. 
Ara Torralba, J.C. (2004). “De Iris a Pepona. Isabel II en el porfolio literario". En Pérez Garzón, J.S. (ed.). Isabel II, los espejos de la reina. Madrid: Marcial Pons, pp. 263-280.

Cano González, A.M. (2002). Un poema desconocíu nel Álbum románticu (18401893) de Dorotea Armiño. Uviéu: Academia de la Llingua Asturiana.

Checa Godoy, A. (2002). Historia de la prensa pedagógica en España. Sevilla: Universidad de Sevilla.

Criado y Domínguez, J. (1889). Literatas españolas del siglo XIX. Apuntes bibliográficos. Madrid: Imprenta de Antonio Pérez Dubrull.

Hartzenbusch, E. (1894). Apuntes para un catálogo de periódicos madrileños desde el año 1661 al 1870. Madrid: Sucesores de Rivadeneyra.

Henestrosa, A. (2009). Alacena de minucias (Introducción y compilación de Adán Cruz Bencomo). México: Porrúa.

Jiménez Morell, I. (1992). La prensa femenina en España (desde sus orígenes a 1868). Madrid: Ediciones de la Torre.

Lledó Patiño, M. (2012). “La visibilidad de las escritoras del XIX en el espacio público de la prensa". Estudios sobre el mensaje periodístico, vol. 18, pp. 569-575.

Lorenzo Benavente, J.B. (1996). “Eulalia de Llanos y Noriega, una poetisa olvidada y pre-cinematográfica" En El Comercio, 4 de marzo de1996.

Martínez Torrón, D. (2008). Poetas románticas españolas (Antología). Madrid: SIAL Ediciones.

Mayoral, M. (1990). Escritoras románticas españolas. Madrid: Fundación Banco Exterior.

Miralles, E. (coord.) (2006). Nueve poetas malogrados del romanticismo español. Barcelona: PPU.

Muñiz Martín, M.E. (1979). Eulalia de Llanos. Madrid: Ediciones Páramo.

Muñiz Martín, M.E. (1990). "Robustiana Armiño, poeta y periodista gijonesa". El Comercio, 17 de enero de 1990. Incluido en Armiño, R. (1990) Dramas de la costa (prólogo de Luis Alberto Prieto García). Candás: Instituto de Bachillerato de Candás, p. 16.

Palacio Valdés, A. (1918): Obras Completas (Tomo IX. El cuarto poder). Madrid: Librería de Victoriano Suárez.

Parada, D.I. (1881). Escritoras y eruditas españolas. Apuntes y noticias, Madrid: M. Minuesa.

Rivas Arranz, L. (2013). Matilde Cherner, salmantina en el olvido. Biografía de una escritora. (Recuperado de http:// historiasdelcuartodeatras.blogspot. com.es/2013/02/matilde-cherner-salmantina-en-el-olvido.html).
Rodríguez Sánchez, M.Á. (2000). “Matilde Cherner: una voz femenina y crítica ante la prostitución en la España de 1880". En Sevilla Arroyo, F. y Alvar, C. (coords.) Actas del XIII Congreso Internacional de Hispanistas (vol. II). Madrid: Castalia, pp. 370-378.

Sánchez Llama, I. (ed.). (2001). Antología de la prensa periódica isabelina escrita por mujeres (1843-1894). Cádiz: Universidad de Cádiz.

Sánchez Llama, I. (ed.). (2002). Galería de escritoras isabelinas. La prensa periódica entre 1833-1895. Madrid: Cátedra.

Servén, C. (2008). "Las escritoras españolas en la prensa infantil hacia 1870". En Fernández, P. y Ortega, M.L. (eds.). La mujer de letras o la letraherida. Discursos y representaciones sobre la mujer escritora en el siglo XIX. Madrid: Consejo Superior de Investigaciones Científicas, pp. $409-425$.

Simón Palmer, M.C. (1991). Escritoras españolas del siglo XIX. Manual bio-bibliográfico. Madrid: Castalia.

Suárez, C. (1959). Escritores y artistas asturianos. Índice bio-bibliográfico (vol. 1). Madrid: Gráficas Summa. 


\section{APÉNDICE. La obra de Robustiana Armiño}

La obra de Robustiana Armiño en la prensa periódica

1841-1848 (viviendo todavía en Gijón)

-Gobierno representativo y constitucional del Bello Sexo español (1841), mensual.

-El Nalón, periódico de literatura, ciencias y artes, Oviedo (1842, dura apenas 5 meses, con Plácido Jove y Hevia, Ruiz de Aguilera...). Hubo tres El Nalón: el 1ㅇ (1842) cofundado por Plácido Jove; en el 3o Laverde y Robustiana Armiño.

-El Eco del Comercio, Madrid (1 poema).

-El Anfión Matritense (2 poemas).

-El Pensamiento, periódico de literatura, ciencias y artes, dedicado al Liceo de Badajoz con Grassi y Coronado (1844).

-El Guadiana, periódico Hispano-Lusitano, Artístico e Industrial. Badajoz (6 poemas).

-EI Sin Nombre; El Pensil del Bello Sexo, suplemento de la revista El Genio, de Víctor Balaguer, (3 poemas, 1845).

-El Vergel de Andalucía, Cordoba (4 poemas);

-El Defensor del Bello Sexo, periódico de literatura, moral ciencias y moda (1845-1846), publica por entregas Las virtudes capitales.

1848-1859 (viviendo en Salamanca)

-El Eco del Comercio, Madrid, (1 poema);

-La Primavera, de Madrid, dirigido por Plácido Jove y Hevia (1 poema).

-La Luna, de Madrid.(2 poemas en 1848).

-La Luneta, Madrid (1 poema).

-Los hijos de Eva (1849), Alicante (luego Madrid), semanario de literatura, ciencias y artes, dirigido por Ventura Ruiz Aguilera y Agustín Mendía (3 poemas de Robustiana Armiño y colaboraciones de Carolina, Avellaneda...). Ruiz Aguilera (1820-1881) que era salmantino, como los Cherner -Matilde y Juan Cuesta-, estaba desterrado en Alicante.

-La llusión (de Miguel Ortiz), periódico de ciencias, literatura, bellas artes y modas, dedicado al bello sexo (18491850).

-El Asturiano (1850).

-El Ovetense; El Fomento de Asturias (ambos en Archivo General de Gijón).

-Ellas. Órgano oficial del Bello Sexo, editado (dirigido) por Alicia Pérez de Gascuña (4 poemas, 1851).

-El Gijonés; El Avisador; El Álbum de la Juventud (con Laverde), 1853.

-El Centinela de Asturias (y su suplemento quincenal El Nalón); El Independiente y El Industrial (ambos de Protasio González Solís, 1829-1908); La Verdad (1854).

-El Faro asturiano (el más importante de los periódicos de Protasio González Solís y uno de los más importantes de España, diario desde 1860 (su suplemento literario, Revista de Asturias); en 1859, colabora Laverde; Revista Universal (1856)

-La Linterna Mágica, Madrid, 1859 (2 textos, periodístico y narrativo). 
18601880 (viviendo en Madrid)

-El Eco de Gijón ; La Aurora de la Vida -9 textos periodísticos (1861)

-Crónica gijonesa (1863)

- Los Ecos del Auseva. Revista de Caridad, Amenidad, Instrucción, Moralidad, Beneficencia (a partir del número 26, La Familia), fundado y dirigido por R. A. ("Sobre la comunicación a Robustiana Armiño de Cuesta de la suscripción de la Reina [Isabel II] por doce ejemplares al periódico semanal Ecos del Auseva que dirige aquella, para su conservación en la Biblioteca Particular" (Oficio 1864-08-10). Según consta en Patrimonio Nacional, Real Biblioteca (accesible internet).

-Almanaque de las Asturias (1864), luego Revista Cántabro-Asturiana, en cuyo №1 colabora Robustiana Armiño con Laverde.

-La Educanda (1865).

-La Guirnalda, periódico quincenal dedicado al Bello Sexo (1867-1870).

-La Moda Elegante Ilustrada (1870) (Robustiana Armiño, Sinués, Avellaneda...).

-Altar y Trono (1 texto periodístico); La Margarita (2 textos periodísticos, 1871).

-El Correo de la Moda, Madrid, dirigida por Ángela Grassi (14 textos periodísticos y 2 poemas en los años 1872 , $1873,1877,1878)$.

-Los Niños. Revista de educación y recreo, Madrid, dirigida por Carlos Frontaura. (9 textos didácticos; traducciones de Grimm). Otros colaboradores: Quintana, Ossorio y Bernard, Hartzenbusch, Carolina Coronado, García Gutiérrez, Arnao, Caballero de Rodas... (1872-1873)

En el № 10 de La Correspondencia de los Niños, R. A. se presenta como autora de "Revista de las Niñas", inserta en el periódico.

-El Periódico para todos. Novelas, viajes, literatura, historia, causas célebres, chistes etc..etc. Semanario Ilustrado editado por Jesús Graciá, y escrito por D. M. Fernández y González, D. R. Ortega y Frías y D. T. Tarrago y Mateos (70 textos periodísticos de R. A entre 1872-1874, algunos de ellos traducciones del inglés y del francés).

-La Mariposa, dirigida por Ermelinda Ormaeche y Begoña.

-La Primera Edad, Madrid (4 textos periodísticos y 1 poema, 1873).

-La Moda Elegante, Madrid (2 textos periodísticos).

-La Iberia, Madrid (1 texto periodístico, 1875).

-Revista Cántabro-Asturiana (con Laverde, 1877).

-La llustración Gallega y Asturiana, publicación por entregas, del № 1 al 36 (excepto 19, 30 y 34), de la novela de R. A. Dramas de la costa.;

-La llustración de la Infancia, Madrid (1878).

-La llustración de los Niños, Madrid (1 texto periodístico y 1 poema en francés).

-La Madre de Familia, Granada (1879).

-La Academia, Madrid.

\section{Obras de Robustiana Armiño}

-Poesías, Oviedo, Martínez Hermanos,1851 (2 vol.)

-Flores del Paraíso o llustración de la infancia, Gijón, Imprenta y librería a cargo de L. González, 1852, un tomo en 8o de XVI y 245 pp. 
-Fotografías sociales, Madrid, 1861, dos tomos en 4 ㅇ․ .

-Las virtudes capitales. Contra soberbia humildad, Madrid, Pedro Montero,1865 (por entregas en El Defensor del Bello Sexo, 1845-1846).

-El ángel de los tristes (A la Reina Isabel II), Madrid, 1864.

-La culpa va en el castigo, Imp. Sres.Martínez y Bogo, Madrid, 1864.

-Dramas de la costa, publicada por entregas en La llustración Gallega y Asturiana, 1879. Edición moderna a cargo de Luis Alberto Prieto García, Instituto de Bachillerato de Candás, Asturias, 1990.

\section{Obras colectivas}

-Corona poética de Manuel José Quintana, en La España musical y literaria, Madrid, 1855. (Un poema de Robustiana Armiño). Otra "corona", en la misma fecha, fue editada por Rivadeneyra.

-Corona poética a la rendición de Tetuán, ed. de Miguel Arcas y Sánchez, Madrid, 1860, Imp. calle de Peralta, num.8, a cargo del mismo editor. En 8o, XVI-152 pp. (Odas: Mạ Josefa Massané de González. Sonetos: Faustina Sáez de Melgar. Romances: Mạ del Pilar Sinués de Marco. Himnos: R. Armiño de Cuenca).

-Poesías dedicadas a Isabel II, Madrid, 1865 (“Circundada de altísimas montañas...”, pp. 9-12.

-Escenas de la vida (colección de cuentos y cuadros de costumbres por una sociedad de autores), 3, segunda serie, Imprenta de R. Labajos, Madrid, 1866. (Incluye el cuento de Robustiana Armiño "La calumnia", así como textos de Ventura de la Vega, Hartzenbusch, Pereda, A. Trueba...

-Corona fúnebre, dedicada a la Reina María de las Mercedes. Madrid-Barcelona, 1878 (“Ay, era una esperanza..”, p. 5). 